\title{
COHOMOLOGY OF FIBRE SPACES WITH GROUP BUNDLE COEFFICIENTS
}

\author{
M. V. MIELKE
}

\begin{abstract}
The purpose of this paper is to construct a resolution of a group bundle $\gamma$, a classifying spectrum for a cohomology functor $H^{*}(; \gamma)$ (coefficients in $\gamma$ ) defined on a category of fibre spaces, and to clarify and note some implications of the close relationship between these two constructions.
\end{abstract}

1. Introduction. A group bundle, as used in this paper, consists of two Hausdorff $k$-spaces $E(\gamma)$ (the total space) and $B$ (the base space), a continuous projection $E(\gamma) \rightarrow B$, and a fibre preserving map from the fibre product (in the category of $k$-spaces) $\gamma \times \gamma$ to $\gamma$ that induces on each fibre of $\gamma$ an abelian group structure. Further, the 0 -section and the inversion map are required to be continuous (see [2], [3], [4]). This notion of group bundle differs from the usual notion in that forming fibre products in the sense of $k$-spaces allows for an increase in the number of group bundle structures on $\gamma$. If, for example, $\gamma$ is locally compact the two notions coincide. Define $H^{p}(B ; \gamma)=H^{p}(B ; \tilde{\gamma})$ where $\tilde{\gamma}$ is the sheaf of germs of sections of $\gamma$ and call $\gamma$ acyclic if $\tilde{\gamma}$ is acyclic (see [1]).

2. Definitions and remarks. An exact sequence of group bundles $0 \rightarrow \gamma_{0} \rightarrow v_{0} \rightarrow^{i_{1}} v_{1} \rightarrow \cdots$ (over a fixed base $B$ ) is a resolution of $\gamma_{0}$ if the associated sequence of sheaves $0 \rightarrow \tilde{\gamma}_{0} \rightarrow \tilde{v}_{0} \rightarrow \cdots$ is exact. The resolution is acyclic if each $\nu_{n}$ is acyclic. By $\left[1\right.$, p. 34], $H^{n}\left(B ; \gamma_{0}\right) \simeq H^{n}\left(C\left(\tilde{\gamma}_{0}\right)\right)$ where $C\left(\tilde{\gamma}_{0}\right)$ is the cochain complex of sheaf sections: $0 \rightarrow \Gamma\left(\tilde{\nu}_{0}\right) \rightarrow \Gamma\left(\tilde{\nu}_{1}\right) \rightarrow \cdots$ associated to an acyclic resolution of $\gamma_{0}$. Define $\gamma_{n}$ to be the image of $i_{n}$. Since $C\left(\tilde{\gamma}_{0}\right)$ is isomorphic to the complex of bundle sections

and

$$
C\left(\gamma_{0}\right): 0 \rightarrow \Gamma\left(v_{0}\right) \stackrel{\Gamma\left(i_{1}\right)}{\longrightarrow} \Gamma\left(v_{1}\right) \rightarrow \cdots
$$

$$
\operatorname{Ker}\left(\Gamma\left(i_{n+1}\right)\right)=\Gamma\left(\gamma_{n}\right),
$$

there is a natural isomorphism $H^{n}\left(B ; \gamma_{0}\right) \simeq \Gamma\left(\gamma_{n}\right) / \operatorname{Im} \Gamma\left(i_{n}\right)$. This result can be interpreted as

2.1. $H^{n}\left(B ; \gamma_{0}\right)$ is naturally isomorphic to the group of equivalence

Received by the editors October 24, 1972.

AMS (MOS) subject classifications (1970). Primary 55B20; Secondary 14F05.

Key words and phrases. Cohomology, group bundle, resolution, spectra. 
classes of sections of $\gamma_{n}$, where two sections are identified if and only if their difference lifts (by $\left.i_{n}\right)$ to a section of $v_{n-1}\left(i_{0}=0\right)$.

Further, the sequence $0 \rightarrow \gamma_{n} \rightarrow v_{n} \rightarrow v_{n+1} \rightarrow \cdots$ is clearly an acyclic resolution, hence

2.2. $H^{p}\left(B ; \gamma_{n}\right) \simeq H^{p}\left(C\left(\gamma_{n}\right)\right) \simeq H^{p+n}\left(B ; \gamma_{0}\right)$ to $(n \geqq 0, p>0)$.

3. An acyclic resolution. A group bundle is said to be an $(L) N D R$ group bundle if (locally) the 0 -section is a vertical deformation retract of an open neighborhood; see [3], [4, 6.2]. In [3] the following is proved.

LEMMA. If $\gamma_{0}$ is an $(L) N D R$ group bundle, then there is an exact sequence of group bundles (depending functorially on $\gamma_{0}$ ).

3.1. $0 \rightarrow \gamma_{0} \rightarrow^{i} \nu_{0} \rightarrow^{j} \gamma_{1} \rightarrow 0$ for which

3.2. $\gamma_{1}$ is an $(L) N D R$ group bundle.

3.3. $j: E\left(v_{0}\right) \rightarrow E\left(\gamma_{1}\right)$ has local sections, i.e. there is an open cover $\left\{U_{\alpha}\right\}$ of $E\left(\gamma_{1}\right)$ and maps $s_{\alpha}$ such that $j s_{\alpha}=\mathrm{id}$ on $U_{\alpha}$.

3.4. $v_{0}$ is shrinkable to the 0-section.

3.5. $j$ has the covering homotopy property for vertical homotopies $H_{t}: X \rightarrow E\left(\gamma_{1}\right)$ if $\gamma_{0}$ is NDR (LNDR if $X$ is paracompact).

Property 3.2 implies a long exact sequence $0 \rightarrow \gamma_{0} \rightarrow v_{0} \rightarrow^{i_{1}} \nu_{1} \rightarrow \cdots$ can be constructed inductively by applying the lemma to $\gamma_{1}$ and splicing the resulting sequence 3.1 to the sequence 3.1 associated to $\gamma_{0}$, etc. Since " " is left exact (by construction [3] $\gamma_{0}$ has topology induced by i) and $\tilde{j}$ is onto by 3.3 (lift germs of sections of $\gamma_{1}$ by an appropriate $s_{\alpha}$ ) the sequence so obtained is a resolution, hereafter called the canonical resolution.

If $B$ is paracompact 3.4 implies $\tilde{\nu}_{0}$ is soft (thus acyclic, [1, p. 49], i.e., every section $s$ over a closed set $K$ extends to $B$. Indeed, there is an open set $U \supset K$ and $\sigma^{\prime} \in \Gamma\left(v_{0} \mid U\right)$ such that $\tilde{\sigma}^{\prime} \mid K=s$. Define $\sigma \in \Gamma\left(v_{0}\right)$ by

$$
\begin{aligned}
\sigma(b) & =0 & & \text { if } \tau(b) \leqq \frac{1}{2}, \\
& =H_{2 \tau(b)-1}\left(\sigma^{\prime}(b)\right) & & \text { if } \tau(b) \geqq \frac{1}{2},
\end{aligned}
$$

where $H_{t}$ is a vertical homotopy shrinking $v_{0}$ (3.4) $\left(H_{0}=0, H_{1}=\mathrm{id}\right)$, $\tau: B \rightarrow I$ is such that $\tau^{-1}(1) \supset \bar{U}_{1}, \tau \mid(B-U)=0$ where $U_{1}$ is open and $U \supset$ $\bar{U}_{1} \supset U_{1} \supset K$. Clearly $\sigma\left|U_{1}=\sigma^{\prime}\right| U_{1}$, thus $\tilde{\sigma}$ extends $s$. This proves

3.6. THEOREM. Any LNDR group bundle over a (paracompact) $k$-space has a (acyclic) resolution.

Assume $B$ is paracompact for the rest of this section. The construction of this resolution implies that

$$
0 \rightarrow \gamma_{n-1} \rightarrow v_{n-1} \stackrel{i_{n}}{\longrightarrow} \gamma_{n} \rightarrow 0
$$

satisfies 3.4, and 3.5 if $\gamma_{0}$ is $L N D R$. This gives a "homotopic" description 
of the "algebraic" equivalence relation on sections of $\gamma_{n}$ (2.1); namely, for $s_{0}, s_{1} \in \Gamma\left(\gamma_{n}\right)$ there is $s \in \Gamma\left(v_{n-1}\right)$ such that $i_{n} s=s_{1}-s_{0}$ if and only if $s_{0}$ and $s_{1}$ are vertically homotopic. Indeed, if $i_{n} s=s_{1}-s_{0}$ define the homotopy by $H_{t}^{\prime}(b)=i_{n}\left(H_{t}(s(b))\right)+s_{0}(b)$ where $H_{t}$ is a shrinking of $v_{n-1}\left(H_{0}=0\right.$, $\left.H_{1}=\mathrm{id}\right)$. Conversely, given an $H_{t}^{\prime}\left(H_{0}^{\prime}=s_{0}, H_{1}^{\prime}=s_{1}\right)$ then $H_{t}^{\prime \prime}=H_{t}^{\prime}-s_{0}$ is a vertical homotopy between 0 and $s_{1}-s_{0}$ such that $H_{0}^{\prime \prime}$ is covered by the 0 -section of $v_{n-1}$. By 3.5, $H_{1}^{\prime \prime}$ is covered by a section $s$ and $i_{n} s=s_{1}-s_{0}$, thus

3.7. If $\gamma_{0}$ is $L N D R$ there is a natural isomorphism between $H^{n}\left(B ; \gamma_{0}\right)$ and the group of equivalence classes of vertically homotopic sections of $\gamma_{n}$.

4. A classifying spectrum. For $B$ a $k$-space let $P_{B}$ be the category of paracompact $k$-fibre spaces ([2], [3]) over $B\left(\xi \in P_{B}\right.$ means $E(\xi)$ is a paracompact $k$-space). For $\gamma_{0}$ a group bundle on $B$ define $H^{p}\left(\xi ; \gamma_{0}\right)$ ( $p$ th cohomology of $\xi$ with coefficients in $\left.\gamma_{0}\right)$ as $H^{p}\left(E(\xi) ; \xi\left(\gamma_{0}\right)\right.$ ) where the group bundle $\xi\left(\gamma_{0}\right)$ is the pullback (in category of $k$-spaces) of $\gamma_{0}$ via the projection $P(\xi)$ of $\xi$. It is not hard to see that conditions 3.1-3.5 are invariant under pullback (the pullback by $P(\xi)$ of 3.1 is an exact sequence on $E(\xi)$ satisfying 3.2-3.5). Consequently the pullback of the canonical resolution of $\gamma_{0}$ is an acyclic resolution of $\xi\left(\gamma_{0}\right)$. Since pullback induces an isomorphism between $\Gamma\left(\xi\left(v_{n}\right)\right)$ and $\operatorname{Hom}\left(\xi, v_{n}\right)$ (the set of fibre preserving maps $\left.\xi \rightarrow v_{n}\right), 2.1$ implies

4.1. If $\gamma_{0}$ is an $L N D R$ then $H^{*}\left(\xi ; \gamma_{0}\right)$ is the cohomology of the complex $\left\{\operatorname{Hom}\left(\xi, v_{n}\right), \operatorname{Hom}\left(i_{n+1}\right)\right\}$.

Result 3.7 (with $\left(E(\xi) ; \xi\left(\gamma_{n}\right)\right)$ replacing $\left.\left(B ; \gamma_{0}\right)\right)$ plus the fact that pull back induces an isomorphism between the group of equivalence classes of vertically homotopic sections of $\xi\left(\gamma_{n}\right)$ and the group of equivalence classes of vertically homotopic fibre maps $\xi \rightarrow \gamma_{n}$, denoted by $\left[\xi, \gamma_{n}\right]$, gives

4.2. THEOREM. For $\gamma_{0}$ an LNDR the image sequence $\left\{\gamma_{1}, \gamma_{2}, \cdots\right\}$ of the canonical resolution of $\gamma_{0}$ is a classifying spectrum for the functor $H^{*}\left(; \gamma_{0}\right)$ defined on $P_{B}$, i.e., $H^{n}\left(\xi ; \gamma_{0}\right) \simeq\left[\xi, \gamma_{n}\right], n>0$.

The following is proved in [3].

4.3. If $\gamma_{0}$ is $N D R$ then $\left[\xi, \gamma_{1}\right]$ and the group of isomorphism classes of numerable principal $\gamma_{0}$ bundles on $\xi$ are naturally isomorphic. Normality of $E(\xi)$ is not needed, but if $E(\xi)$ is paracompact, numerable can be omitted and $N D R$ replaced by $L N D R$. A similar interpretation holds for $\left[\xi, \gamma_{n}\right]$.

Combining 4.2, 2.2 and 4.3 gives

4.4. THEOREM. If $\gamma_{0}$ is LNDR then $H^{p}\left(\xi ; \gamma_{n}\right)$ is naturally isomorphic to the group of isomorphism classes of principal $\gamma_{p+n-1}$ bundles on $\xi$ for $\xi \in P_{B}(n \geqq 0, p>0)$. 
Besides providing a geometric interpretation of $H^{*}\left(; \gamma_{0}\right)$ this implies $(p=1)$ that $\gamma_{n}$ is the classifying group bundle (see [3]) of $\gamma_{n-1}$ (actually

$$
E\left(v_{n}\right) \stackrel{i_{n}}{\longrightarrow} E\left(\gamma_{n}\right) \rightarrow B
$$

is a universal bundle for $\left.\gamma_{n-1}\right)$.

\section{REFERENCES}

1. G. Bredon, Sheaf theory, McGraw-Hill, New York, 1967. MR 36 \#4552.

2. A. Grothendieck, A general theory of fibre spaces with structure sheaf, NSF Research Project Report No. 4, University of Kansas, Lawrence, Kan., 1958.

3. M. Mielke, Universal fibre bundles of group bundles (to appear).

4. N. E. Steenrod, A convenient category of topological spaces, Michigan Math. J. 14 (1967), 133-152. MR 35 \#970.

Department of Mathematics, University of Miami, Coral Gables, Florida 33124 